\title{
Practical scheme to share a secret key through a quantum channel with a $27.6 \%$ bit error rate
}

\author{
H. F. Chau* \\ Department of Physics, University of Hong Kong, Pokfulam Road, Hong Kong, China
}

(Received 13 May 2002; published 20 December 2002)

\begin{abstract}
A secret key shared through quantum key distribution between two cooperative players is secure against any eavesdropping attack allowed by the laws of physics. Yet, such a key can be established only when the quantum channel error rate due to eavesdropping or imperfect apparatus is low. Here, a practical quantum key distribution scheme by making use of an adaptive privacy amplification procedure with two-way classical communication is reported. Then, it is proven that the scheme generates a secret key whenever the bit error rate of the quantum channel is less than $0.5-0.1 \sqrt{5} \approx 27.6 \%$, thereby making it the most error resistant scheme known to date.
\end{abstract}

DOI: 10.1103/PhysRevA.66.060302

PACS number(s): 03.67.Dd, 89.20.Ff, 89.70.+c

Quantum key distribution (QKD) is the process of sharing a secret bit string, known as the key, between two cooperative players, commonly called Alice and Bob, by exchanging quantum signals. Since an unknown quantum state cannot be perfectly cloned [1,2], any eavesdropping attempt by Eve will almost surely disturb the transmitted quantum states. Thus, by carefully estimating the error rate of the transmitted quantum states, Alice and Bob know with great confidence the quantum channel error rate, which in turn reflects the eavesdropping rate. (In contrast, Alice and Bob can never be sure if Eve has eavesdropped in classical key distribution because classical signals can be copied without being caught in principle.) If the estimated eavesdropping rate is high, they abort the scheme and start all over again. On the other hand, if the estimated eavesdropping rate is low, privacy amplification procedure such as quantum error correction or entanglement purification can be used to distill out an almost perfectly secure key [3-5].

It is instructive to devise a secure QKD scheme that tolerates as high a quantum channel error rate as possible and subject that scheme to a vigorous cryptanalysis. Indeed, Mayers [5] and Biham et al. [6] proved the security of the so-called Bennett-Brassard 1984 (BB84) QKD scheme [7] against all kinds of attack allowed by the laws of quantum physics. Following Mayers' proof, a provably secure key is established whenever the channel error rate is less than about $7 \%$. Lo and Chau proved the security of an entanglementbased QKD scheme [3]. By scrambling the qubits before transmission and using the quantum Gilbert-Varshamov argument for a general quantum stabilizer code [8], the Lo and Chau scheme tolerates up to about $18.9 \%$ channel error. Nonetheless, the Lo and Chau scheme requires quantum computers and hence is not practical at the present moment. By properly combining the essences of the Mayers as well as Lo and Chau proofs, Shor and Preskill gave an ingenious security proof of the BB84 scheme that applies up to $11.0 \%$ channel error [9]. The most error resistant QKD scheme known to date was recently found by Gottesman and Lo. Built upon the Shor-Preskill proof, Gottesman and Lo showed that a carefully designed privacy amplification pro-

*Electronic address: hfchau@hkusua.hku.hk cedure with two-way communication increases the error tolerant level of a QKD scheme. In particular, they proved that the six-state QKD scheme introduced by Bruss [10] tolerates up to about $23.7 \%$ bit error rate (or equivalently up to about $35.5 \%$ channel error rate) [11]. Recently, Gottesman and Lo further improved their two-way communication protocol and showed that it generates a provably secure key up to $26.4 \%$ bit error rate [12]. (Here, the channel error rate and bit error rate refer to the rate of quantum and spin-flip errors occurring in the insecure noisy quantum channel, respectively.)

Here, I report an adaptive privacy amplification procedure for the six-state scheme. Then, I prove that this procedure enables the six-state scheme to generate a provably secure key up to $0.5-0.1 \sqrt{5} \approx 27.6 \%$ bit error rate (or equivalently up to $0.75-0.15 \sqrt{5} \approx 41.4 \%$ quantum channel error), breaking the $26.4 \%$ bit error rate record of Gottesman and Lo. This scheme is also practical, requiring no quantum computer or search for asymptotically good quantum codes. Since no BB84-based scheme can tolerate more than $25 \%$ bit error rate [12], the $27.6 \%$ bit error rate tolerable six-state scheme reported here convincingly demonstrates the advantage in error tolerability of the six-state scheme over BB84.

Before reporting the adaptive procedure, let me briefly review the privacy amplification procedure introduced by Gottesman and Lo [11]. In the first step of the Gottesman-Lo privacy amplification procedure, Alice and Bob perform entanglement purification with local quantum operation and two-way classical communication (LOCC2 EP). Specifically, they randomly pair up their corresponding bits in the string and compare the result of a bilateral exclusive or (BXOR) in each pair. They keep their corresponding control bits in each pair only if their parities agree. In the second step, Alice and Bob apply the $[3,1,3]_{2}$ phase error correction (PEC). This is equivalent to randomly forming trios of the remaining bits and replacing each trio by their corresponding parities [11]. Alice and Bob apply LOCC2 EP and PEC alternatively until the error rate of the resultant signal can be handled by an asymmetric Calderbank-Shor-Steane (CSS) quantum code $[13,14]$ with great confidence. Then, they apply the ShorPreskill error correction procedure [9] to the remaining bits using the above CSS code. By doing so, they end up sharing a secret key with exponentially close to $100 \%$ confidence. 
Gottesman and Lo further showed that their procedure brings down the error rate whenever the channel error rate is less than about $23.7 \%$ [11].

The Gottesman-Lo two-way privacy amplification procedure reviewed above can be improved in two ways. First, there is no reason why one must apply LOCC2 EP and PEC alternately. Instead, Alice and Bob should devise a suitable privacy amplification procedure based on the estimated $\sigma_{x}$, $\sigma_{y}$, and $\sigma_{z}$ error rates of the qubits transmitted through the insecure noisy channel. Besides, they may use $[r, 1, r]_{2}$ for some $r>3$ as their phase error correction code. In fact, using this approach, Gottesman and Lo proved that the six-state scheme can tolerate a bit error rate up to $26.4 \%$ [12]. Second, although the asymmetric CSS code used by Gottesman and Lo is known to exist using Gilbert-Varshamov type of argument [13], explicitly finding that it may be difficult, in general. Fortunately, concatenated quantum CSS code is already sufficient in handling the final error correction in the privacy amplification procedure. More importantly, various concatenated quantum CSS codes and their decoding algorithms are known.

Before I report my six-state scheme, I first call upon two propositions below to study the effects of LOCC2 EP and PEC on the error rates of the signal.

Proposition 1. Suppose Alice sends Bob several qubits through a quantum channel whose $\sigma_{x}, \sigma_{y}$, and $\sigma_{z}$ error rates due to either noise or eavesdropping are $p_{x}, p_{y}$, and $p_{z}$, respectively. Let $p_{I}=1-p_{x}-p_{y}-p_{z}$. If the error suffered by each qubit is independent of the other then the error rates of the resultant qubits after going through one around of LOCC2 EP are given by

$$
\begin{aligned}
& p_{I}^{\mathrm{EP}}=\frac{p_{I}^{2}+p_{z}^{2}}{\left(p_{I}+p_{z}\right)^{2}+\left(p_{x}+p_{y}\right)^{2}}, \\
& p_{x}^{\mathrm{EP}}=\frac{p_{x}^{2}+p_{y}^{2}}{\left(p_{I}+p_{z}\right)^{2}+\left(p_{x}+p_{y}\right)^{2}}, \\
& p_{y}^{\mathrm{EP}}=\frac{2 p_{x} p_{y}}{\left(p_{I}+p_{z}\right)^{2}+\left(p_{x}+p_{y}\right)^{2}}, \\
& p_{z}^{\mathrm{EP}}=\frac{2 p_{I} p_{z}}{\left(p_{I}+p_{z}\right)^{2}+\left(p_{x}+p_{y}\right)^{2}} .
\end{aligned}
$$

Furthermore, the error rate in each of the resultant qubit after the LOCC2 EP is independent of each other.

Proof. Recall that in the LOCC2 EP, Alice and Bob randomly pair up their corresponding shares of the qubits and apply BXOR to each pair. During the BXOR operation, any $\sigma_{x}$ error in the control qubit remains unaltered. In contrast, the $\sigma_{z}$ error of the resultant control qubit is inherited from both the original control and the target qubits [15]. Since Alice and Bob reject the pair if the measurement results of their share of target qubit differ, hence the remaining control qubit is error-free if the error operator acting on the original control and target qubits equal $I \otimes I$ or $\sigma_{z} \otimes \sigma_{z}$. Similarly, the remaining control qubit suffers $\sigma_{x}, \sigma_{y}$, and $\sigma_{z}$ errors if the error operator acting on the original control and target qubits equal $\sigma_{x} \otimes \sigma_{x}$ or $\sigma_{y} \otimes \sigma_{y}, \sigma_{x} \otimes \sigma_{y}$ or $\sigma_{y} \otimes \sigma_{x}$, and $I \otimes \sigma_{z}$ or $\sigma_{z} \otimes I$, respectively. Since error suffered by each qubit is independent of the other, hence Eq. (1) holds. The independence of resultant error rates after the LOCC2 EP procedure follows directly from the independence of channel error for the qubits received by Bob.

By Proposition 1 and mathematical induction, it is straight forward to check that the error rates of the resultant qubits after going through $k$ rounds of LOCC2 EP are given by

$$
\begin{aligned}
& p_{I}^{k \mathrm{EP}}=\left[\left(p_{I}+p_{z}\right)^{2^{k}}+\left(p_{I}-p_{z}\right)^{2^{k}}\right] / 2 D, \\
& p_{x}^{k \mathrm{EP}}=\left[\left(p_{x}+p_{y}\right)^{2^{k}}+\left(p_{x}-p_{y}\right)^{2^{k}}\right] / 2 D, \\
& p_{y}^{k \mathrm{EP}}=\left[\left(p_{x}+p_{y}\right)^{2^{k}}-\left(p_{x}-p_{y}\right)^{2^{k}}\right] / 2 D, \\
& p_{z}^{k \mathrm{EP}}=\left[\left(p_{I}+p_{z}\right)^{2^{k}}-\left(p_{I}-p_{z}\right)^{2^{k}}\right] / 2 D,
\end{aligned}
$$

where $D=\left(p_{I}+p_{x}\right)^{2^{k}}+\left(p_{x}+p_{y}\right)^{2^{k}}$. So whenever $p_{I}>1 / 2$, $p_{I}^{k \mathrm{EP}}>1 / 2$, and $p_{z}^{k \mathrm{EP}}<1 / 2$. Further, $p_{I}^{k \mathrm{EP}}, p_{z}^{k \mathrm{EP}} \rightarrow 1 / 2$ and $p_{x}^{k \mathrm{EP}}, p_{y}^{k \mathrm{EP}} \rightarrow 0$ as $k \rightarrow \infty$. That is, repeated application of LOCC2 EP reduces $\sigma_{x}$ and $\sigma_{y}$ errors at the expense of possibly increasing $\sigma_{z}$ and perhaps also the overall error rates.

Proposition 2. We use the notations in Proposition 1. Suppose Alice and Bob divide their shared pairs into $n$ sets each containing $r$ shared pairs. And then they perform one round of PEC using the $[r, 1, r]_{2}$ majority vote phase error correction code. The resultant error rates of the signal after one round of PEC satisfy

$$
\begin{gathered}
p_{x}^{\mathrm{PEC}}+p_{y}^{\mathrm{PEC}} \leqslant r\left(p_{x}+p_{y}\right), \\
p_{y}^{\mathrm{PEC}}+p_{z}^{\mathrm{PEC}} \leqslant\left[4\left(p_{I}+p_{z}\right)\left(p_{x}+p_{y}\right)\right]^{r / 2} \leqslant e^{-2 r\left(0.5-p_{z}-p_{y}\right)^{2}},
\end{gathered}
$$

provided that $p_{I}>1 / 2$. Also, the error rate in each of the resultant qubit after PEC is independent of each other.

Proof. The idea of the proof is the same as that in Proposition 1 . Recall that the error syndrome of the $[r, 1, r]_{2}$ phase error correction code is given by

$$
\left[\begin{array}{ccccc}
1 & 1 & & & \\
1 & & 1 & & \\
\vdots & & & \ddots & \\
1 & & & & 1
\end{array}\right]
$$

So, after measuring this error syndrome, the $\sigma_{z}$ error stays on the control qubit while the $\sigma_{x}$ error propagates from the control as well as all target qubits to the resultant control qubit [15]. Therefore, upon PEC, the resultant control qubit is spin-flip error-free whenever there is an even number of qubits amongst the $r$ of them in the same set suffering spin-flip error. Hence, the first inequality in Eq. (3) holds. Similarly, the resultant control qubit suffers from phase-shift error provided that at least $[(r-1) / 2\rceil$ out of the $r$ qubits are suffering 
from phase-shift error. Such a probability of occurrence equals $\sum_{a \geqslant[(r-1) / 2]}\left(\begin{array}{c}r \\ a\end{array}\right)\left(p_{y}+p_{z}\right)^{a}\left(p_{I}+p_{x}\right)^{r-a}$. Combining with the inequality [16]

$$
\begin{aligned}
\sum_{k=0}^{\lambda n}\left(\begin{array}{l}
n \\
k
\end{array}\right) p^{k}(1-p)^{n-k} \leqslant & \lambda^{-\lambda n}(1-\lambda)^{-(1-\lambda) n} \\
& \times p^{\lambda n}(1-p)^{(1-\lambda) n}
\end{aligned}
$$

for $0<\lambda<p$, we conclude that the probability of having a phase error is upper bounded by $\left[4\left(p_{I}+p_{x}\right)\left(p_{y}+p_{z}\right)\right]^{r / 2}$. Thus, the first line of the second inequality in Eq. (3) is satisfied. To arrive at the second line, one simply considers the Taylor-series expansion of $\ln \left[1+\left(2 p_{I}+2 p_{x}-1\right)\right]+\ln [1$ $\left.+\left(2 p_{y}+2 p_{z}-1\right)\right]$ and uses the observation that all odd power terms in the expansion are canceled.

Proposition 2 tells us that if $0.5-p_{z}-p_{y} \gg \sqrt{p_{x}+p_{y}}$, the phase error can be greatly reduced after one round of PEC by choosing $r \approx 0.01 /\left(p_{x}+p_{y}\right)$. Specifically, with this choice of $r$, Eq. (3) implies that $p_{y}^{\mathrm{PEC}}+p_{z}^{\mathrm{PEC}}$ is exponentially small while $p_{x}^{\mathrm{PEC}}+p_{y}^{\mathrm{PEC}}$ is at most about $1 \%$.

Alice and Bob may exploit the dynamics of LOCC2 EP and PEC to perform their privacy amplification. Specifically, they first repeatedly apply LOCC2 EP until $0.5-p_{z}-p_{y}$ $\gg \sqrt{p_{x}+p_{y}}$. Then, applying PEC once will bring the overall error rate $p_{x}+p_{y}+p_{z}$ down to an acceptable value. And then, Alice and Bob may choose to use the concatenated Steane's seven-qubit code in the Shor-Preskill procedure. Recall that Steane's seven-qubit code corrects one error out of seven qubits [14]. Thus, as long as Alice and Bob randomly permute the bits before applying the Shor-Preskill procedure, the overall error rate that is almost surely tolerated by the concatenated Steane's seven-qubit code is equal to the smallest positive root of the equation

$$
1-\lambda=(1-\lambda)^{7}+7(1-\lambda)^{6} \lambda
$$

namely, about $5.8 \%$. The upshot is that the error correction algorithm for the concatenated Steane's seven-qubit code is known and can be carried out efficiently.

With these two improvements in mind, I write down my modified six-state scheme below.

(1) Alice prepares $N$ qubits each randomly chosen from $|0\rangle,|1\rangle,|0\rangle \pm|1\rangle$, and $|0\rangle \pm i|1\rangle$ and sends them to Bob [10]. Bob acknowledges the reception of the qubits and measures each of them randomly and independently along one of the following three bases: $\{|0\rangle,|1\rangle\},\{|0\rangle \pm|1\rangle\}$, and $\{|0\rangle$ $\pm i|1\rangle\}$. Then, Alice and Bob publicly announce the bases they have used to prepare or measure each qubit. They keep only those qubits that are prepared and measured in the same basis.

(2) Alice and Bob estimate the channel error rate by sacrificing a few qubits. Specifically, they divide the qubits into three sets according to their bases of measurement. They randomly pick $O(\ln [1 / \epsilon])$ qubits from each set and publicly compare the preparation and measurement results of each chosen qubit. In this way, they know the estimated channel error rate with standard deviation $\epsilon$. (A detailed proof of this claim can be found in Ref. [4].) If the estimated channel error rate is too high, they abort the scheme and start all over again.

(3) Using the convention that $|0\rangle,|0\rangle-|1\rangle$, and $|0\rangle$ $-i|1\rangle$ represent a logical 0 while the $|1\rangle,|0\rangle+|1\rangle$, and $|0\rangle+i|1\rangle$ represent a logical 1 , Alice and Bob convert their untested measured qubits into secret strings. Then, they perform the following privacy amplification procedure on their secret bit strings.

(a) They apply the LOCC2 EP procedure proposed by Gottesman and Lo in Ref. [11]. Specifically, they randomly pair up their corresponding secret bits and announce the parities of each pair. They keep the control bit in each pair only if their announced parities for the pair agree. They repeat the above LOCC2 EP procedure until there is an integer $r>0$ such that the estimated quantum channel error given by Eq. (3) is less than 5\%. They abort the scheme either when such an integer $r$ is greater than the number of remaining bits they have or when they have used up all their bits in this procedure.

(b) They apply the PEC procedure introduced by Gottesman and Lo in Ref. [11] using the $[r, 1, r]_{2}$ majority vote phase error correction code once. Specifically, Alice and Bob randomly divide the resultant bits into sets each containing $r$ bits. They replace each set by the parity of the $r$ bits in the set.

(c) Alice and Bob randomly permute the order of their remaining bits and apply the Shor-Preskill privacy amplification procedure [9] to these bits with the concatenated Steane's seven qubit code. The level of concatenation depends on the estimated worst case $p_{x}+p_{y}+p_{z}$ given by Eq. (3) and the final required fidelity of the state. Specifically, suppose that the concatenated Steane's seven qubit code is constructed from two binary classical codes $C_{1}$ and $C_{2}$ satisfying $C_{2} \subset C_{1}$. Alice randomly picks a codeword $u \in C_{1}$ and publicly announces the sum of $u$ and her remaining bit string modulo 2. Bob subtracts Alice's announced bit string from his own remaining bit string modulo 2; and then he applies the $C_{2}$ error correction to recover the codeword $u \in C_{1}$. They use the coset $u+C_{2}$ as their secret key.

To prove the security of the above scheme, I follow the arguments of Refs. [3,9,11,17]. First, since this is a prepareand-then-measure scheme, any Eve's quantum cheating strategy can be reduced to a classical one $[3,17]$. Second, Eve does not know how Alice and Bob group the qubit pairs in LOCC2 EP and PEC beforehand. Hence, the resultant error rate after going through either LOCC2 EP or PEC depends only on the probabilities of $\sigma_{x}, \sigma_{y}$ and $\sigma_{z}$ errors and the number of qubits transmitted [3,11]. Thus, to study the asymptotic error tolerable rate of the above scheme, it suffices to consider cheating strategies characterized only by $p_{x}, p_{y}$ and $p_{z}$ respectively. Since Alice chooses the six states randomly and uniformly, the untested qubits can be regarded as having passed through a depolarizing channel [11]. Hence, Alice and Bob almost surely know that $p_{x}=p_{y}=p_{z}$ for their untested qubits.

From Eq. (3) in Proposition 2, I know that after applying LOCC2 EP $\mathrm{k}$ times, PEC will bring the quantum error rate down to, say, 5\% if $r=0.04 /\left(p_{x}^{k \mathrm{EP}}+p_{y}^{k \mathrm{EP}}\right)$ and $2 r(0.5$ 
$\left.-p_{z}^{k \mathrm{EP}}-p_{y}^{k \mathrm{EP}}\right) \gg 1$. Putting $p_{x}=p_{y}=p_{z}=\left(1-p_{I}\right) / 3$ into Eq. (2), I conclude that this is possible when $k \rightarrow \infty$ and $\left(p_{I}\right.$ $\left.-p_{z}\right)^{2}>\left(p_{I}+p_{z}\right)\left(p_{x}+p_{y}\right)$. This condition implies that $20 p_{I}^{2}-10 p_{I}-1>0$ or $p_{I}>0.25+0.15 \sqrt{5}$. In other words, the above scheme tolerates a bit error rate up to $p_{x}+p_{y}$ $=0.5-0.1 \sqrt{5} \approx 27.6 \%$ (which corresponds to a quantum channel error rate of $p_{x}+p_{y}+p_{z}=0.75-0.15 \sqrt{5} \approx 41.4 \%$ ).

Besides, once Alice and Bob estimate the channel error rates, then they can efficiently compute the number of LOCC2 EP to be applied as well as the level of concatenation for the Steane's seven qubit code to be used. Finally, the error syndrome of the concatenated Steane's seven-qubit code as well as the corresponding Shor-Preskill procedure are straight forward to compute.

The $27.6 \%$ bit error rate bound reported here shows that the six-state scheme is more noise resistant than the BB84 scheme since no BB84 scheme can tolerate more than $25 \%$ bit error [12]. In addition, the adaptive privacy amplification idea can be applied to increase the error tolerant level in a number of QKD schemes. For instance, the above adaptive privacy amplification procedure enables the BB84 to generate a provably secure key whenever the bit error rate is less than $20.0 \%$ (or equivalently, a quantum channel error rate of less than 39.9\%). Besides, one can show the existence of a biased entanglement-based QKD scheme requiring quantum computers, whose key is provably secure whenever the bit error rate is less than $33.3 \%$ [18].

This work was financially supported in part by the HKU and the RGC Grant No. HKU 7095/97P of the HKSAR government. I would like to thank H.-K. Lo for explaining his work with Gottesman on applying two-way classical communication to quantum key distribution and for discussing with me his recent work with Gottesman [12] prior to its public dissemination, D. Gottesman and H.-K. Lo for their valuable discussions on privacy amplification, and D. W. C. Leung and H.-K. Lo for their critical reading of this manuscript.
[1] W.K. Wootters and W. Zurek, Nature (London) 299, 802 (1982).

[2] D. Dieks, Phys. Lett. A 92, 271 (1982).

[3] H.-K. Lo and H.F. Chau, Science 283, 2050 (1999), as well as the supplementary material available at http:// www.sciencemag.org/feature/data/984035.shl

[4] H.-K. Lo, H.F. Chau, and M. Ardehali, e-print quant-ph/0011056v2.

[5] D. Mayers, J. Assoc. Comput. Mach. 48, 351 (2001); see also his preliminary version in D. Mayers, in Proceedings of Crypto'96 (Springer- Verlag, Berlin, 1996), pp. 343-357.

[6] E. Biham, M. Boyer, P.O. Boykin, T. Mor, and V. Roychowdhury, in Proceedings of the 32nd Annual ACM Symposium on Theory of Computing (STOC2000) (ACM Press, New York, 2000), pp. 715-724.

[7] C.H. Bennett and G. Brassard, in Proceedings of the IEEE International Conference on Computers, Systems and Signal
Processing, Bangalore, India (IEEE, New York, 1984), pp. 175-179.

[8] A.R. Calderbank, E.M. Rains, P.W. Shor, and N.J.A. Sloane, IEEE Trans. Inf. Theory 44, 1369 (1998).

[9] P.W. Shor and J. Preskill, Phys. Rev. Lett. 85, 441 (2000).

[10] D. Bruss, Phys. Rev. Lett. 81, 3018 (1998).

[11] D. Gottesman and H.-K. Lo, e-print quant-ph/0105121.

[12] H.-K. Lo (private communications).

[13] A.R. Calderbank and P. Shor, Phys. Rev. A 54, 1098 (1996).

[14] A.M. Steane, Proc. R. Soc. London, Ser. A 452, 2551 (1996).

[15] M.A. Nielsen and I.L. Chuang, Quantum Computation and Quantum Information (Cambridge University Press, Cambridge, London, 2000), Chap. 10.

[16] S. Roman, Coding And Information Theory (Springer, Berlin, 1992), Eq. (1.2.5), p. 26.

[17] H.-K. Lo, Quant. Inform. Comp. 1(2), 81 (2001).

[18] H.F. Chau (unpublished). 